\title{
The Range of a Random Walk on a Comb
}

\author{
János Pach* \\ EPFL, Lausanne, Switzerland \\ and Rényi Institute, Budapest, Hungary \\ pach@cims.nyu.edu
}

\author{
Gábor Tardos ${ }^{\dagger}$ \\ Rényi Institute, Budapest, Hungary \\ tardos@renyi.hu
}

Submitted: July 10, 2013; Accepted: Sep 25, 2013; Published: Oct 7, 2013

Mathematics Subject Classification: 05C81

\begin{abstract}
The graph obtained from the integer grid $\mathbb{Z} \times \mathbb{Z}$ by the removal of all horizontal edges that do not belong to the $x$-axis is called a comb. In a random walk on a graph, whenever a walker is at a vertex $v$, in the next step it will visit one of the neighbors of $v$, each with probability $1 / d(v)$, where $d(v)$ denotes the degree of $v$. We answer a question of Csáki, Csörgő, Földes, Révész, and Tusnády by showing that the expected number of vertices visited by a random walk on the comb after $n$ steps is $\left(\frac{1}{2 \sqrt{2 \pi}}+o(1)\right) \sqrt{n} \log n$. This contradicts a claim of Weiss and Havlin.
\end{abstract}

\section{Introduction}

The theory of finite Markov chains or, in graph-theoretic language, the theory of random walks on graphs is a classical topic in probability theory. It has many applications from flows in networks, through statistical physics to complexity theory in computer science (see Lovász [Lo96] and Woess [Wo00]).

To obtain a random walk in a locally finite graph $G$ with vertex set $V(G)$, we start at any vertex $v \in V(G)$ and in the next step we move to one of its neighbors, independently of all previous events, with probability $1 / d(v)$. Here, $d(v)$ denotes the number of edges in $G$ incident to $v$. Every neighbor of $v$ is equally likely to come next. Perhaps the simplest example is a random walk on the $d$-dimensional integer grid $\mathbb{Z}^{d}$, studied by Pólya [Po21]. He proved that for $d=1$ and 2 , with probability 1 , a random walk will return to its starting point infinitely often, while for $d \geqslant 3$ only a finite number of times.

*Supported by NSF Grant CCF-08-30272, by OTKA under EUROGIGA projects GraDR and ComPoSe 10-EuroGIGA-OP-003, and by Swiss National Science Foundation Grants 200021-137574 and $200020-144531$.

†Supported by an NSERC grant, and the OTKA grant NN-102029 
The 2-dimensional comb $\mathbb{C}^{2}$ is a spanning tree of the integer grid $\mathbb{Z}^{2}$ obtained by removing all of its "horizontal" edges (that is, edges parallel to the $x$-axis) that do not belong to the $x$-axis. In this graph, all vertices (or "sites") $(x, y)$ have degree 2 , except for the vertices of the form $(x, 0)$, which have degree 4 . For any nonnegative integer $n$, let $W_{n}=\left(X_{n}, Y_{n}\right)$ be the random variable denoting the position of the walker after $n$ steps. We assume the walk starts at the origin, so $W_{0}=(0,0)$. The study of random walks on the comb was initiated by Weiss and Havlin [WeH86], as a model of "anomalous diffusion on fractal structures." These investigations were later extended to combs of higher dimensions by Gerl [Ge86] and Cassi and Regina [CaR92]. Krishnapur and Peres [KrP04] proved that, on the 2-dimensional comb, with probability 1, two independent walkers meet only a finite number of times. This is a rather surprising phenomenon, in view of the fact that the random walk is recurrent, that is, a single random walker visits each site an infinite number of times with probability 1. Some insight was provided by Bertacchi and Zucca [BeZ03] and by Bertacchi [Be06], whose asymptotic estimates suggested that a walker spends most of her time moving vertically along a "tooth" of the comb. Several strong approximation and limit theorems for random walks on a comb have been established by Csáki, Csörgö, Földes, and Révész [CsCs09, CsCs11].

Let $V_{n}$ denote the number of vertices (sites) visited during the first $n$ steps of the random walk $W_{n}=\left(X_{n}, Y_{n}\right)$ on the 2-dimensional comb. According to the main result in [WeH86], the expected value of $V_{n}$ is asymptotically proportional to $n^{3 / 4}$, for large $n$. It is not hard to see that almost surely the deviation of the horizontal projection $X_{n}$ of the walk is roughly $n^{1 / 4}$, while the expected length of the vertical projection is of order $n^{1 / 2}$. See, e.g., Bertacchi [Be06] (cp. Panny and Prodinger [PaP85]). This suggests that the expected number of sites visited by the random walk on $\mathbb{C}^{2}$ is around $n^{1 / 4} \cdot n^{1 / 2}=n^{3 / 4}$, as was stated by Weiss and Havlin [WeH86]. The aim of this note is to show that the truth is closer $n^{1 / 2}$, than to $n^{3 / 4}$.

All logarithms used in this paper are natural logarithms.

Theorem 1 The expected value of $V_{n}$, the number of vertices visited during the first $n$ steps of a random walk on the 2-dimensional comb, satisfies

$$
E\left[V_{n}\right]=\left(\frac{1}{2 \sqrt{2 \pi}}+o(1)\right) \sqrt{n} \log n
$$

\section{Elementary properties of a random walk on $\mathbb{Z}$}

We collect some well-known and easy facts about 1-dimensional random walks on $\mathbb{Z}$; all of them can be found, e.g., in [Fe68] or [Ré07]. For any pair of integers $n, i \geqslant 0$, let $p_{n, i}$ denote the probability that starting at 0 , after $n$ steps we end up at the vertex (integer) $i$. We have

$$
p_{n, i}=\left(\begin{array}{c}
n \\
\frac{n+i}{2}
\end{array}\right) \frac{1}{2^{n}},
$$


where the value of the above binomial coefficient is considered 0 , whenever $\frac{n+i}{2}$ is not an integer. It follows that

$$
p_{n, i} \leqslant\left(\begin{array}{c}
n \\
\lfloor n / 2\rfloor
\end{array}\right) \frac{1}{2^{n}}=\left(\sqrt{\frac{2}{\pi}}+o(1)\right) \frac{1}{\sqrt{n}}
$$

as $n$ tends to infinity. Moreover, we have

$$
p_{n, i}=\left(\sqrt{\frac{2}{\pi}}+o(1)\right) \frac{1}{\sqrt{n}}
$$

whenever $i / \sqrt{n} \rightarrow 0$ and $n+i$ is even.

Let $A_{n}$ stand for the number of times the random walk visits the origin during the first $n$ steps and $B_{n}$ for the number of sites visited during the the first $n$ steps. We have

$$
\begin{gathered}
E\left[A_{n}\right]=\sum_{m=0}^{n} p_{m, 0}=\left(\sqrt{\frac{2}{\pi}}+o(1)\right) \sqrt{n} \\
E\left[B_{n}\right]=\left(2 \sqrt{\frac{2}{\pi}}+o(1)\right) \sqrt{n} .
\end{gathered}
$$

See, e.g., [Ré07], p. 253. Finally, for any $j>0$, let $r_{j}$ denote the probability that starting at position 0 , the infinite random walk on $\mathbb{Z}$ reaches $j$ before it would return to 0 . We have

$$
r_{j}=\frac{1}{2 j} .
$$

\section{Proof of Theorem 1}

The vertices and edges of the comb $\mathbb{C}^{2}$ that belong to the $x$-axis form the backbone. The connected components of the graph obtained from $\mathbb{C}^{2}$ after the removal of the backbone are called teeth.

We consider the projections $\left(X_{i}\right)$ and $\left(Y_{i}\right)$ of the two-dimensional walk $\left(X_{i}, Y_{i}\right)$, separately. First, we reduce these one-dimensional walks by getting rid of the steps when the value does not change. In this way, horizontal steps contribute only to the reduced projection $\left(X_{i}^{\prime}\right)$ and vertical steps contribute only to the reduced projection $\left(Y_{i}^{\prime}\right)$. Note that with probability 1 both reduced walks are infinite and they are distributed as the standard random walk on the line. Let us consider the random walk on the comb up to (and including) the $n$th vertical move. We call this walk $W$. Its reduced projections are $\left(X_{i}^{\prime}\right)_{i=0}^{a}$ and $\left(Y_{i}^{\prime}\right)_{i=0}^{n}$, where the random variable $a$ is the number of horizontal moves in $W$. Let $c$ stand for the number of sites on the backbone reached by $W$. It is easy to describe the asymptotic behavior of $E[a]$ and $E[c]$. 


\section{Lemma 2}

$$
\begin{gathered}
E[a]=\left(\sqrt{\frac{2}{\pi}}+o(1)\right) n^{1 / 2} \\
E[c]=\Theta\left(n^{1 / 4}\right)
\end{gathered}
$$

Proof: Let $d$ denote the number of times $W$ moves from a tooth to a position on the backbone, including the starting position at the origin, but not including the final position after the last move, even if it satisfies this condition. Clearly, $d$ is distributed as $A_{n-1}$. $W$ has $d$ chances to make horizontal moves. At each chance, it makes precisely $i$ consecutive horizontal moves with probability $2^{-i-1}$. Thus, for the expected value of $a$, the number of horizontal moves in $W$, we have

$$
E[a]=E\left[d \sum_{i=0}^{\infty} i 2^{-i-1}\right]=E[d]=E\left[A_{n-1}\right]=\left(\sqrt{\frac{2}{\pi}}+o(1)\right) \sqrt{n}
$$

Here the last equality follows by (2).

Obviously, the conditional distribution of $c$, given $a$, is the same as the distribution of $B_{a}$, so that by (3) we obtain

$$
E[c \mid a]=\left(2 \sqrt{\frac{2}{\pi}}+o(1)\right) \sqrt{a}
$$

Hence, we have

$$
E[c]=\left(2 \sqrt{\frac{2}{\pi}}+o(1)\right) E[\sqrt{a}] \leqslant\left(2 \sqrt{\frac{2}{\pi}}+o(1)\right) \sqrt{E[a]}=\left(\frac{2^{7 / 4}}{\pi^{3 / 4}}+o(1)\right) n^{1 / 4} .
$$

On the other hand, the limiting distribution of $d / \sqrt{n}$ (i.e., that of $A_{n-1} / \sqrt{n}$ ) is equal to the distribution of the absolute value of a random variable with standard normal distribution (see, e.g., [Ré07], p. 506). Therefore, we have $P(d / \sqrt{n} \geqslant 1 / 2) \geqslant 1 / 2+o(1)$. Using the fact that, given $d$, the inequality $a \geqslant d$ holds with probability exactly $1 / 2$, we obtain that $E[\sqrt{a}] \geqslant(1 / 4+o(1)) \sqrt{\frac{1}{2} \sqrt{n}}=\Theta\left(n^{1 / 4}\right)$. In view of $(5)$, this implies the second part of the lemma.

We prove Theorem 1 by estimating the expected number of sites $V_{n}^{\prime}$ reached by $W$. As $W$ makes $n+a$ moves, it reaches the $V_{n}$ sites visited in the first $n$ steps and potentially at most $a$ further sites. According to the first part of Lemma 2, this potential increase is so small that it does not change the asymptotic behavior of the expected value.

First, we establish the upper bound. We classify a site $(i, j)$ as close if $|j|<n^{1 / 4}$, far if $|j|>2 n^{1 / 2} \log ^{1 / 2} n$, and intermediate if $n^{1 / 4} \leqslant|j| \leqslant 2 n^{1 / 2} \log ^{1 / 2} n$, and estimate the expected number of sites reached in each class, separately. 
The number of close sites reached is less than $2 n^{1 / 4}+1$ times the number $c$ of sites reached on the backbone. Thus, by Lemma 2, the expected number of close sites reached is $O(\sqrt{n})$. The number of far sites reached can easily be bounded by the number of steps $0 \leqslant i \leqslant n$ with $\left|Y_{i}^{\prime}\right|>2 n^{1 / 2} \log ^{1 / 2} n$. For each $i \leqslant n$, the Chernoff bound gives $P\left(\left|Y_{i}^{\prime}\right|>2 n^{1 / 2} \log ^{1 / 2} n\right)<1 / n$. Therefore, the expected number of far sites reached is at most 1.

Let us call the tooth of the comb containing the site where $W$ ends the final tooth. In case $Y_{n}^{\prime}=0$, there is no final tooth. The total number of intermediate sites on the final tooth is less than $n^{1 / 2} \log ^{1 / 2} n$, so this also bounds the expected number of intermediate sites reached on the final tooth.

Finally, we consider the number $w$ of intermediate sites reached outside the final tooth. When such a site at distance $j$ is reached, it must be reached through a vertical move. If the $i$-th vertical move reaches it for the last time in $W$, then we have $\left|Y_{i}^{\prime}\right|=j$ and the part of the walk after the $i$-th vertical move (starting from this site) must reach the backbone before it comes back to the same site. The probability for $\left|Y_{i}^{\prime}\right|=j$ is $2 p_{i, j}$. Assuming that this happens, according to (4), the probability that the infinite random walk reaches the backbone before it returns to the same site is $r_{j}=1 /(2 j)$. Hence, the total probability is at most $p_{i, j} / j$. We bound $E[w]$ by summing $p_{i, j} / j$ over all $1 \leqslant i \leqslant n$ and $n^{1 / 4} \leqslant j \leqslant 2 n^{1 / 2} \log ^{1 / 2} n$. Using the fact that $p_{i, j}=0$ if $i+j$ is odd and, by (1), $p_{i, j} \leqslant(\sqrt{2 / \pi}+o(1)) / \sqrt{i}$, it follows that

$$
E[w] \leqslant \sum_{j} \sum_{i} p_{i, j} / j \leqslant\left(\sqrt{\frac{2}{\pi}}+o(1)\right) \sum_{\substack{i, j \\ i+j \text { even }}} \frac{1}{j \sqrt{i}} \leqslant\left(\sqrt{\frac{2}{\pi}}+o(1)\right) \frac{1}{4} \sqrt{n} \log n .
$$

Summing over all the sites reached by $W$, we obtain the upper bound in the theorem:

$$
E\left[V_{n}\right] \leqslant E\left[V_{n}^{\prime}\right] \leqslant\left(\frac{1}{2 \sqrt{2 \pi}}+o(1)\right) \sqrt{n} \log n .
$$

Before turning to the lower bound, we introduce the symbol $u_{j}$ to denote the probability that $W$ reaches the site $(0, j)$. We need the following lemma.

\section{Lemma 3}

$$
u_{j}=O\left(\frac{n^{1 / 4} \log n}{|j|+1}\right)
$$

Proof: Let $t_{i}$ stand for the probability that $W$ reaches the site $(i, 0)$. Clearly, we have $E[c]=\sum_{i=-\infty}^{+\infty} t_{i}$. Let $W^{\prime}$ denote the random walk on the comb starting at the origin and ending with the $2 n$-th vertical move. For the number $V_{2 n}^{\prime}$ of the sites $W^{\prime}$ reached, we have

$$
E\left[V_{2 n}^{\prime}\right]=O(\sqrt{n} \log n)
$$

by the upper bound (6) we have just proved. For every $i$ and every $j$, the probability that $W^{\prime}$ reaches the site $(i, j)$ is at least $t_{i} u_{j}$. Indeed, with probability $t_{i}$ the walk reaches 
$(i, 0)$ before the $n$-th vertical move, and after that it reaches $(i, j)$ within $n$ further vertical steps with probability $u_{j}$. Note that if $(i, j)$ is reached, then at least $|j|+1$ sites of the form $\left(i, j^{\prime}\right)$ are reached (along the same tooth). Therefore, we obtain

$$
E\left[V_{2 n}^{\prime}\right] \geqslant \sum_{i}(|j|+1) t_{i} u_{j}=(|j|+1) u_{j} E[c] .
$$

From here, applying our upper bound (7) and Lemma 2, the result follows.

Now we are ready to prove the lower bound in Theorem 1.

Let $Z_{i}$ stand for the site reached by the $i$-th vertical move of the random walk $W$. Let $q_{i, j}$ stand for the probability that $\left|Y_{i}^{\prime}\right|=j$ and $Z_{i}$ is reached by the $i$-th vertical move for the last time in $W, 0<i \leqslant n$. Each site that does not belong to the backbone and is reached by $W$, is reached for the last time by a single uniquely determined vertical move. Thus, we have

$$
E\left[V_{n}^{\prime}\right]=E[c]+\sum_{0<j, 0<i \leqslant n} q_{i, j} .
$$

Suppose that $Z_{i}$ is not on the backbone. If after the $i$-th vertical move the random walk $W$ returns to the backbone before it would revisit $Z_{i}$, and after returning to the backbone it still does not visit $Z_{i}$ during the following $n$ vertical moves, then $Z_{i}$ was reached in $W$ for the last time by the $i$-th vertical move. Therefore, using the notation in Section 2, we have

$$
q_{i, j} \geqslant 2 p_{i, j} r_{j}\left(1-u_{j}\right) \text { for } 0<i \leqslant n, j>0 .
$$

The lower bound for $E\left[V_{n}^{\prime}\right] \geqslant \sum q_{i, j}$ can be obtained by evaluating the terms $q_{i, j}$. Let us consider only those terms $q_{i, j}$ for which $n^{1 / 4} \log ^{2} n<j<n^{1 / 2} / \log n, \quad j^{2} \log n \leqslant i \leqslant n$, and $i+j$ is even. For these values, by Lemma 3, we have $u_{j}=o(1)$. Since $j / \sqrt{i} \rightarrow 0,(1)$ yields that $p_{i, j}=(\sqrt{2 / \pi}+o(1)) / \sqrt{i}$. Combining this with (4), inequality (8) gives

$$
q_{i, j} \geqslant\left(\sqrt{\frac{2}{\pi}}+o(1)\right) \frac{1}{\sqrt{i j}} .
$$

Thus, for a fixed $j$, we get

$$
\sum_{j^{2} \log n \leqslant i \leqslant n, i \equiv j} q_{i, j} \geqslant\left(\sqrt{\frac{2}{\pi}}+o(1)\right) \frac{\sqrt{n}}{j} .
$$

Summing over all $j, n^{1 / 4} \log ^{2} n<j<n^{1 / 2} / \log n$, we obtain

$$
E\left[V_{n}^{\prime}\right] \geqslant\left(\sqrt{\frac{2}{\pi}}+o(1)\right) \sqrt{n} \sum_{j} \frac{1}{j} \geqslant\left(\frac{1}{2 \sqrt{2 \pi}}+o(1)\right) \sqrt{n} \log n,
$$

as claimed. Note that our estimates for the expectation of $V_{n}^{\prime}$ carry over to the expectation of $V_{n}$, as $\left|V_{n}^{\prime}-V_{n}\right| \leqslant a$ and $E[a]=O(\sqrt{n})$. This completes the proof of Theorem 1 . 


\section{Acknowledgment}

We are indebted to G. Tusnády for calling our attention to the problem addressed in this note, and to E. Csáki, M. Csörgő, A. Földes, and P. Révész for their valuable remarks.

\section{References}

[Be06] D. Bertacchi, Asymptotic behaviour of the simple random walk on the 2dimensional comb, Electron. J. Probab. 11 (2006), no. 45, 1184-1203.

[BeZ03] D. Bertacchi and F. Zucca, Uniform asymptotic estimates of transition probabilities on combs, J. Aust. Math. Soc. 75 no. 3, 325-353.

[CaR92] D. Cassi and S. Regina, Random walks on $d$-dimensional comb lattices, Mod. Phys. Lett. B 6 (1992),1397-1403.

[CsCs09] E. Csáki, M. Csörgö, A. Földes, and P. Révész, Strong limit theorems for a simple random walk on the 2-dimensional comb, Electron. J. Probab. 14 (2009), no. 82, 2371-2390.

[CsCs11] E. Csáki, M. Csörgö, A. Földes, and P. Révész, On the local time of random walk on the 2-dimensional comb, Stochastic Processes and their Applications 121:6 (2011) 1290-1314.

[Fe68] W. Feller, An Introduction to Probability Theory and its Applications. Vol. I. (3rd ed.), John Wiley \& Sons, New York, 1968.

[Ge86] P. Gerl, Natural spanning trees of $\mathbb{Z}^{d}$ are recurrent, Discrete Math. 61 (1986), 333-336.

[KrP04] M. Krishnapur and Y. Peres, Recurrent graphs where two independent random walks collide finitely often, Electron. Comm. Probab. 9 (2004), 72-81

[Lo96] L. Lovász, Random walks on graphs: a survey, in: Combinatorics, Paul Erdős is eighty, Vol. 2, Bolyai Soc. Math. Stud. 2, 353-397, J. Bolyai Math. Soc., Budapest, 1996.

[PaP85] W. Panny and H. Prodinger, The expected height of paths for several notions of height, Studia Sci. Math. Hungar. 20 (1985), no. 1-4, 119-132.

[Po21] G. Pólya, Über eine Aufgabe der Wahrscheinlichkeitsrechnung betreffend die Irrfahrt im Strassennetz, Math. Annalen 84 (1921), 149-160.

[Ré07] A. Rényi, Probability Theory, Dover Publications, Mineola, New York, 2007. Originally published by North-Holland, Amsterdam and Akadémiai Kiadó, Budapest, 1970.

[WeH86] G. H. Weiss and Sh. Havlin, Some properties of a random walk on a comb structure, Physica A 134 (1986), 474-482.

[Wo00] W. Woess, Random walks on infinite graphs and groups, Cambridge Tracts in Mathematics 138, Cambridge University Press, London, 2000. 